\title{
Presencia de la prensa digital española en la Web social: análisis de Menéame
}

\author{
Por Enrique Orduña-Malea y José-Antonio Ontalba-Ruipérez
}
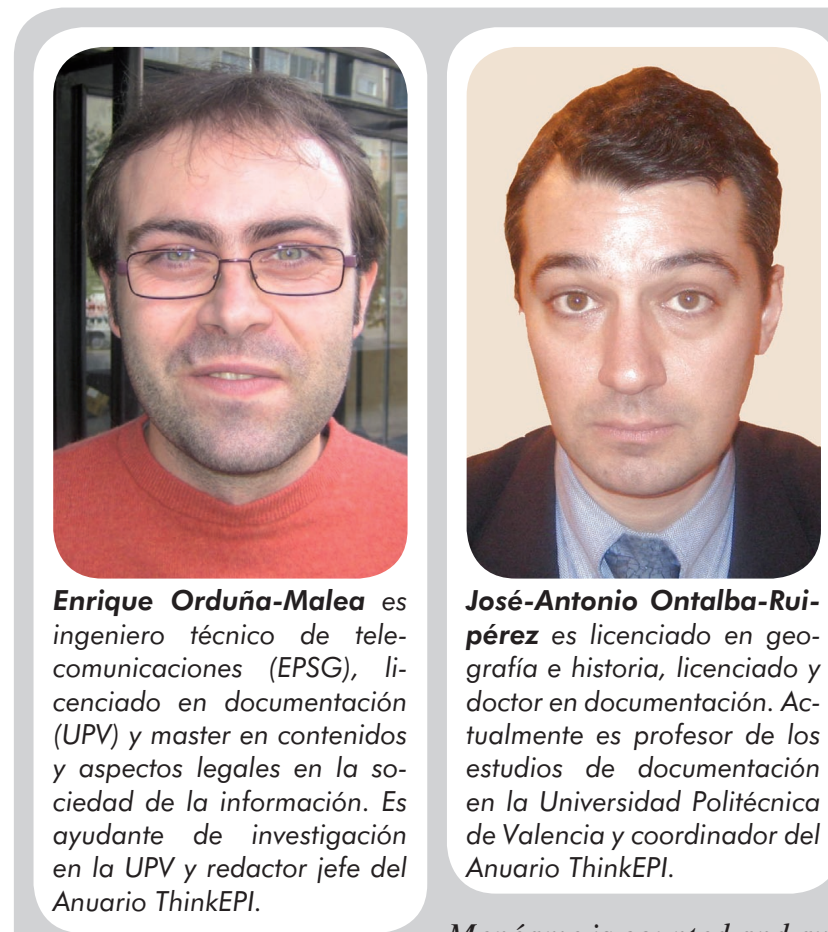

Resumen: El objetivo es medir la visibilidad de la prensa española en Menéame, el gestor social de contenidos más importante en castellano, durante 2007 y el primer trimestre de 2008. A tal fin se contabiliza el número de noticias publicadas en Menéame y se promedia con los votos y comentarios recibidos, centrándose en la evolución de los cinco medios con mayor presencia; finalmente se contrasta el ranking obtenido con el de OJD Interactiva. El medio de prensa más representado es El país, al que le siguen a cierta distancia El mundo y 20minutos y, más alejados, La vanguardia y El periódico de Catalunya.

Palabras clave: Prensa digital, Diarios digitales, Web social, Web 2.0, Participación, Gestor de noticias, Menéame, Espa$\tilde{n} a$.

Title: Presence of the Spanish digital press on the social Web: analysis of Menéame

Abstract: The objective of our study is to measure the visibility of the Spanish press in Menéame, the most important social content manager in Spanish, during 2007 and the first quarter of 2008. To this end, the number of news items published in Menéame is counted and averaged with votes and comments received, focusing on the evolution of the five media with a greater presence; finally, the obtained ranking is contrasted with that of OJD Interactiva. The most represented newspaper is El País, followed at a certain distance by El Mundo and 20minutos and, farther behind, La Vanguardia and El Periódico de Catalunya.

Keywords: Digital press, Digital newspaper, Social Web, Web 2.0, Participation, News manager, Menéame, Spain.

Orduña-Malea, Enrique; Ontalba-Ruipérez, José-Antonio. "Presencia de la prensa digital española en la web social: análisis de Menéame”. En: El profesional de la información, 2008, septiembre-octubre, v. 17, n. 5, pp. 511-518.

DOI: 10.3145/epi.2008.sep.04

\section{Introducción}

Ante la caída continuada en todo el mundo de lectores habituales de prensa escrita y el aumento lento pero progresivo de la diarios en internet, la visibilidad de la prensa en la web y el impacto de ésta en los medios son temas de creciente interés (Salaverría, 2005).

La enorme disponibilidad de información periodística en internet ha dado lugar al desarrollo de servicios de recopilación de noticias, que se clasifican fundamentalmente en bases de datos (Mynews), servicios de clipping (Acceso, Almaclip) y buscadores de noticias, tanto profesionales (iMente, iConoce) como gratuitos (Google news) (Guallar, 2006).

En septiembre de 1997 aparece un nuevo tipo de servicio, representado por Slashdot, donde son los pro- pios usuarios quienes recopilan y estructuran noticias en metadatos para su publicación. La gran influencia de ese promocionador de noticias dió lugar al denominado "efecto Slashdot" por el que se colapsa una web a causa de la cantidad de visitas en poco tiempo redirigidas desde este tipo de servicios cuando difunde una novedad importante (Notess). Su equivalente en España, Barrapunto, aparece en 2000.

\section{http://slashdot.org \\ http://barrapunto.com}

La adaptación de este modelo de comunicación periodística a la web 2.0 se da a finales de 2004 con Digg, un servicio de publicación de noticias por promoción democrática. Es el primer gestor social de noticias. El éxito de este servicio llevó a la publicación de su versión española, Menéame, en diciembre de 2005. 


\section{http://www.digg.com}

http://meneame.net/

A pesar de estar en boga, todos estos servicios no han estado exentos de problemas:

- la propiedad intelectual de algunos contenidos, como el embargo de noticias de pago por parte de $\mathrm{Fi}$ nancial Times (Chillingworth; Eaton),

- la propiedad industrial, como la publicación en Digg de la clave para desencriptar HD DVD ${ }^{1}$,

- o la validez o fiabilidad de las noticias, como la invención de éstas en Google news (Wiggins).

\section{Cómo funciona}

Menéame permite que los usuarios registrados envíen noticias de periódicos digitales, de blogs o de otras fuentes web. En realidad y como ocurre en Digg, no se publica la noticia sino el enlace a la fuente principal. Una vez remitida pasa a un listado de "pendientes" y según los votos que obtenga (se entiende que en función de su interés), podrá pasar a la página principal donde alcanzará una mayor visibilidad y número de visitas.

Tal es el poder de los principales gestores sociales de noticias y el interés de la prensa en luchar por el espacio web que en apenas unos años los medios han pasado de intentar evitar que sus contenidos se difundieran sin su permiso a incluir enlaces en sus noticias para que puedan ser enviadas directamente a los gestores sociales y conseguir así un mayor tráfico.

Menéame es un servicio imitación de Digg programado por Ricardo Galli, el cual ha liberado el software. Este hecho junto al éxito alcanzado en poco tiempo, ha llevado al desarrollo de una gran cantidad de proyectos que se pueden clasificar en clones (servicios que han tomado y adaptado el código fuente a sus necesidades, como por ejemplo DocuMenea), forks (que parten de ese código fuente y posteriormente toman direcciones y objetivos diferentes) y proyectos independientes ${ }^{2}$.

\section{http://www.documenea.com}

En cuanto a éstos destaca Fresqui, que ha superado a Barrapunto en el intervalo estudiado y se acerca a Menéame (figura 1).

\section{http://www.fresqui.com}

\section{Objetivos}

Se advierte en la bibliografía del tema que la mayoría hace referencia a noticias de actualidad y polémicas, básicamente como notas en diferentes blogs. Sobre Digg hay algunos análisis descriptivos y estudios sociológicos del comportamiento de los usuarios (fundamentalmente, Lerman). Pero sobre Menéame no hay ninguna investigación, con la salvedad del estudio de

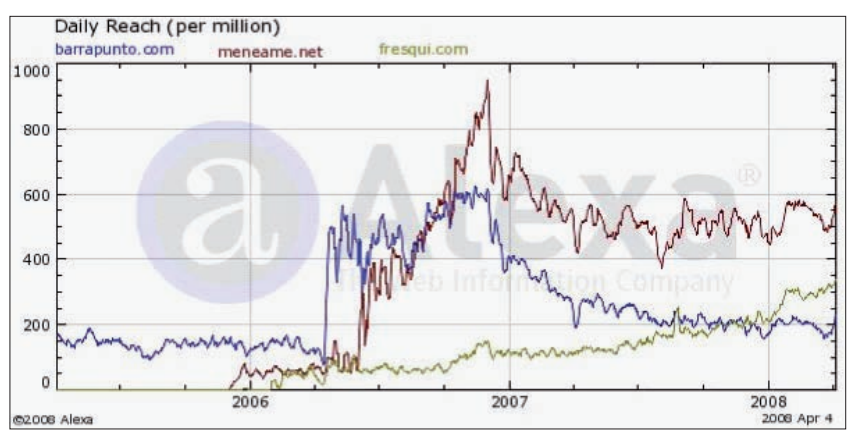

Figura 1. Evolución del tráfico en Barrapunto, Menéame y Fresqui Fuente: Alexa (http://www.alexa.com)

Esciudad.com ${ }^{3}$, y el trabajo del Departamento de Lenguajes y Sistemas Informáticos de la Universidad de Sevilla de análisis de redes de las etiquetas ${ }^{4}$, aparte de los posts estadísticos del blog de Menéame ${ }^{5}$.

El presente trabajo se justifica por tanto ante la necesidad de estudios descriptivos que permitan llevar a cabo investigaciones analíticas y cualitativas y se plantea como objetivo principal medir la presencia y distribución de la prensa española en Menéame, el gestor social de contenidos más importante en castellano. Para ello se analizará:

- la distribución y evolución de las noticias enviadas durante todo 2007 y principios de 2008,

- la identificación de los medios de prensa españoles utilizados, $\mathrm{y}$

- el peso de los principales medios de prensa.

\section{Material y métodos}

Los datos relativos a las noticias publicadas fueron recogidos manualmente durante marzo y abril de 2008 a través de la página de portada de Menéame, donde se archiva la totalidad de noticias enviadas y publicadas desde el inicio de este servicio (la primera noticia publicada data del 7 de diciembre de 2005).

Como se dijo, se capturaron las noticias de 2007, así como del primer cuatrimestre de 2008.

El procedimiento consistió en recoger para cada noticia la fecha en la que había sido publicada, el número de votos y comentarios recibidos y el medio de publicación, para posteriormente decidir si provenía de un medio nacional de prensa digital o no. En el momento de la recogida de datos, las noticias están cerradas: no admiten más votos y los admitidos son positivos.

La razón por la que se ha recogido el número de comentarios y votos es que son indicadores del impacto de la noticia en Menéame, pues supone que la noticia ha tenido algún eco (al margen de que sea negativo o positivo); así, los medios con más votos tienen un mayor impacto en el sistema. 
En cualquier caso, de forma manual se procedió a enlazar con la noticia y comprobar su procedencia. En los casos en los que esto no fue posible (enlaces rotos) la evaluación se realizó a través de la url de la noticia, situada justo debajo del enlace al titular.

Para los objetivos de este trabajo no se consideraron noticias de prensa nacional digital las enviadas desde blogs, medios de prensa extranjeros, ni otros medios de comunicación como páginas web de radio, televisión o portales temáticos. Sin embargo sí que se han tenido en cuenta las agencias de noticias Reuters, Europa Press y $B B C$ Mundo.

De esta misma forma se descartaron las enviadas desde las secciones de blogs de algunos periódicos digitales, pues el objetivo del trabajo era precisamente estudiar la visibilidad de las noticias formales y tradicionales, redactadas por profesionales en medios de prensa.

Tampoco se consideraron las imágenes, viñetas o tiras cómicas, aunque provinieran de un medio nacional. Asimismo quedaron excluidas las informaciones aparecidas en semanarios o revistas.

Todas las noticias no consideradas como prensa nacional digital son tratadas en este trabajo como "otros medios".

Posteriormente se procedió a la descarga, desde la página de OJD Interactiva, del fichero correspondiente a los datos de acceso a los medios digitales durante 2007, es decir: las visitas totales de la web de cada diario con el fin de comparar la distribución de medios obtenida de esta fuente con la de Menéame.

\section{http://ojdinteractiva.ojd.es/comparativas.php}

De todas formas, debe señalarse que en los datos que ofrece $O J D$ (con un nuevo sistema de medición desde principios de 2008), más allá de la problemática acerca de su fiabilidad (Fernández, 2007), no se encuentra la totalidad de medios de prensa detectados en Menéame. Especialmente, no se dispone de los datos relativos a El país y La vanguardia, que no se someten a la auditoría de difusión de $O J D$. En estos dos casos no hay nada que permita comparar con el mismo criterio: así, mientras que El país ha optado por publicar los datos internos obtenidos por la herramienta Site catalyst de Omniture, La vanguardia no ha publicitado el sistema de medición. A título ilustrativo y para completar este vacío se utiliza como referencia también el Estudio General de Medios (EGM) de la Asociación para la Investigación en Medios de Comunicación.

\section{http://www.aimc.es/}

Por lo tanto y teniendo en cuenta que el objetivo de este trabajo es el estudio descriptivo cuantitativo de la presencia de la prensa digital española en Menéame, se utilizará el ranking de OJD Interactiva, que es el más exhaustivo y accesible, y también el EGM para comparar de manera general la distribución de los medios.

\section{Resultados y discusión}

\subsection{Distribución y evolución de noticias de prensa}

El número total de noticias enviadas a Menéame durante el intervalo de muestreo (enero de 2007 a abril de 2008) ha sido de 23.373, de las que $8.505(36,4 \%)$ han sido consideradas procedentes de medios de prensa y el resto $(63,6 \%)$ de "otros medios". Un seguimiento mensual de estos porcentajes muestra un crecimiento de los diarios tradicionales, que pasan del $33 \%$ en enero de 2007 al 43\% en abril de 2008. La media de noticias en el rango estudiado es de 1.461 por mes. La evolución del número total de noticias a lo largo de los meses se puede observar en la figura 2 .

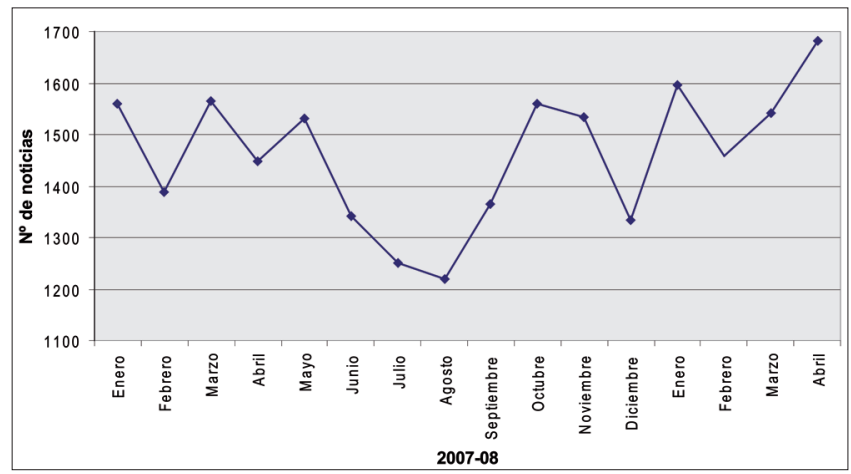

Figura 2. Evolución del número de noticias

Se constata una caída previsible por los períodos vacacionales y muy acusada durante los meses de verano, así como en el mes de diciembre. De igual manera se puede ver cómo durante febrero tanto de 2007 como de 2008 existe un descenso debido al menor número de días del mes. Finalmente se observa un crecimiento notable y continuo en los dos últimos meses.

\subsection{Impacto de las noticias}

En la figura 3 se detalla la evolución del promedio de número de comentarios y de votos por mes que reciben las noticias de prensa.

La gráfica muestra una mayor actividad en Menéa$m e$ con un crecimiento progresivo tanto en el promedio de votos recibidos como en el de comentarios.

En el caso concreto del número de votos, el aumento ha sido notable pues se ha pasado de 257 por noticia a principios de 2007 a situarse sobre los 465 a primeros de 2008.

El crecimiento del promedio de comentarios también ha sido elevado, casi duplicando los valores obtenidos en 2008 respecto de 2007 para los primeros me- 


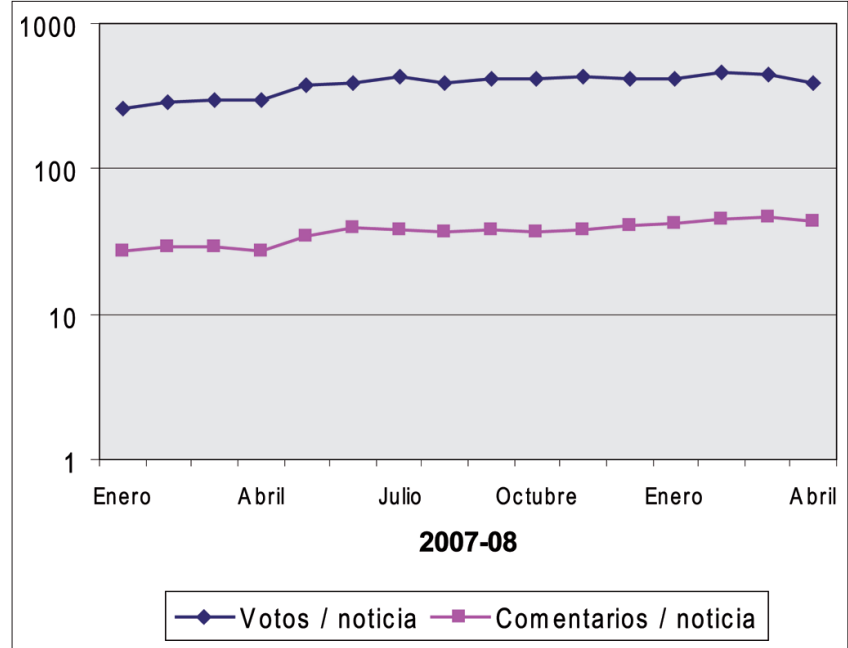

Figura 3. Promedio de votos y comentarios por noticia

ses del año. Se observa un pico de crecimiento situado, como en el caso del número de votos, a principios de verano. Desde finales de 2007 el número de comentarios en promedio presenta un crecimiento constante.

\subsection{Identificación y presencia de los medios de prensa}

Se ha identificado un total de 188 medios de prensa (tabla 1). En la figura 4 se muestra la distribución de los que han superado el centenar, en función del número de noticias publicadas.

Se observa que los seis medios con más presencia (El país, El mundo, 20 minutos, La vanguardia, El periódico y Europa press) representan el 51\% de noticias de prensa publicadas en Menéame.

En el primer cuatrimestre de 2008 El país se mantuvo como el diario digital con mayor representación en Menéame, y El mundo superó a 20 minutos. Por otra parte, $A B C$ y El plural pierden puestos, mientras que Público y $A D N$ presentan subidas importantes.

\subsection{El impacto de los principales medios de prensa}

Ante la dificultad de llevar a cabo un análisis indivi-

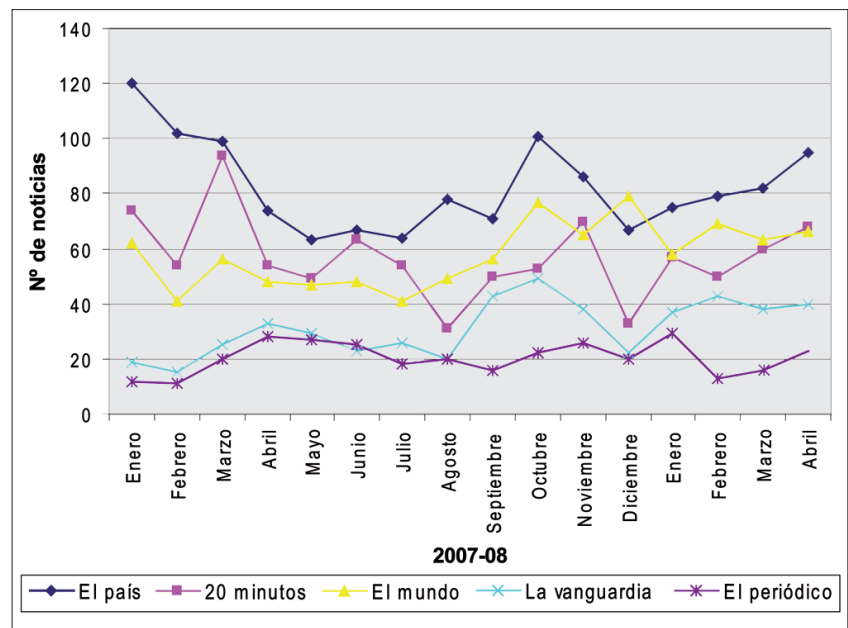

Figura 5. Evolución de noticias publicadas en los cinco medios más representados

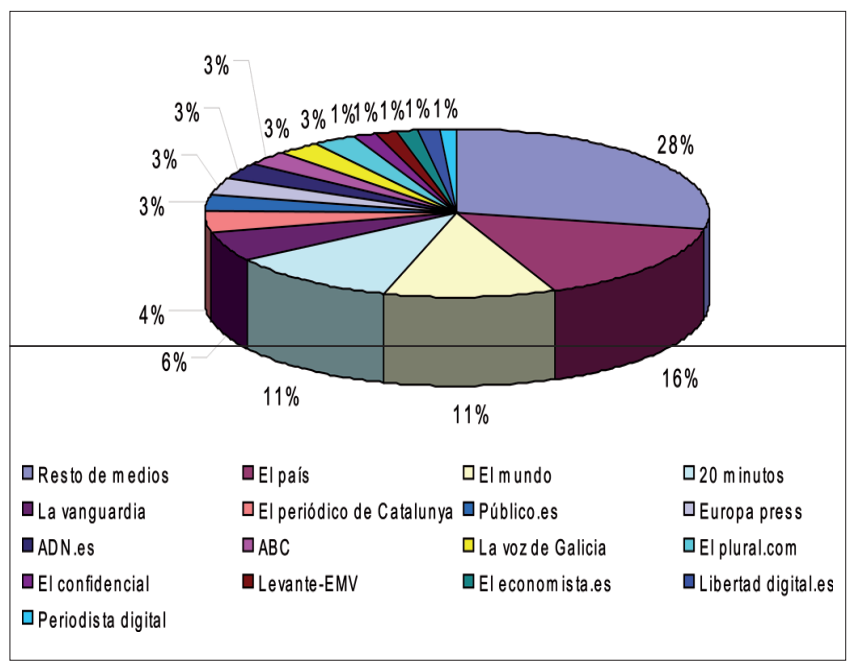

Figura 4. Presencia de los medios de prensa

dualizado por medio, en la figura 5 se muestran los resultados referidos a la evolución de noticias, votos y comentarios de los cinco medios más representados (El país, 20 minutos, El mundo, La vanguardia, El periódico).

Los datos muestran un acercamiento entre los tres principales medios. Es de destacar la existencia de tres grupos: uno formado por El país, que encabeza el ranking en solitario; otro conformado por 20 minutos y $E l$ mundo; y el último, en el que se encuentran La vanguardia y El periódico.

En la figura 6 se comprueba que el promedio de votos tanto positivos como negativos por noticia ha experimentado un crecimiento general importante, pues a principios de 2008 los cinco primeros medios han aumentado en torno al 50\%. Sin embargo en el último mes se detecta una caída generalizada, importantísima en el caso de El mundo. Es constatable la evolución irregular de 20 minutos a lo largo de los meses.

En la figura 7 también se observa una evolución positiva en el promedio de comentarios por noticia para todos los medios. Destaca la subida constante de $\mathrm{La}$

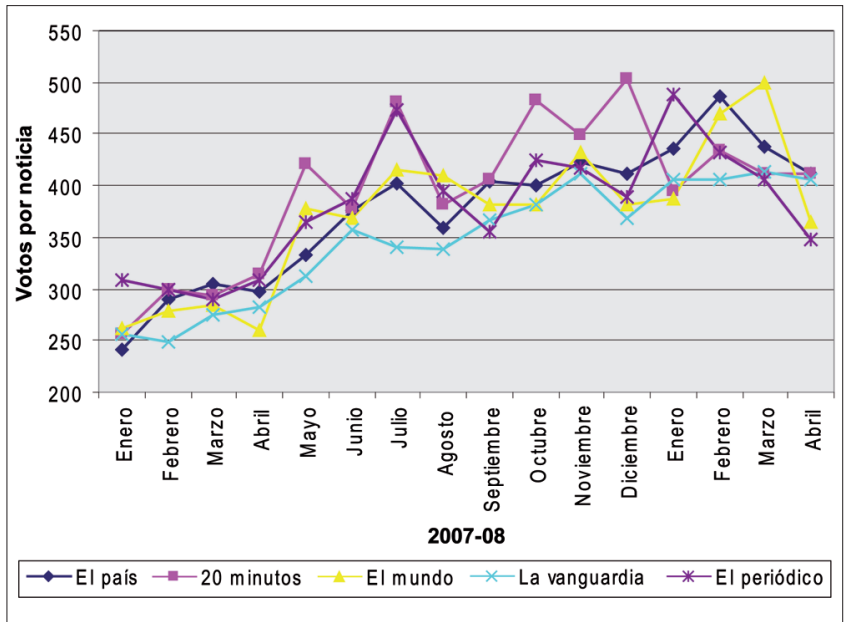

Figura 6. Evolución de votos por noticia en los cinco medios más representados 


\begin{tabular}{|c|c|c|c|c|c|c|c|c|}
\hline $\begin{array}{l}\text { Nú- } \\
\text { me- } \\
\text { ro }\end{array}$ & Medio & $\begin{array}{c}\text { Noti- } \\
\text { cias }\end{array}$ & $\begin{array}{c}\text { Nú- } \\
\text { me- } \\
\text { ro }\end{array}$ & Medio & $\begin{array}{l}\text { Noti- } \\
\text { cias }\end{array}$ & $\begin{array}{c}\text { Nú- } \\
\text { me- } \\
\text { ro }\end{array}$ & Medio & $\begin{array}{l}\text { Noti- } \\
\text { cias }\end{array}$ \\
\hline 1 & El país.es & 1.323 & 64 & La Rioja.com & 17 & \begin{tabular}{|l|}
127 \\
\end{tabular} & Valéncia hui & 3 \\
\hline 2 & El mundo & 925 & 65 & Eco diario.es & 16 & 128 & Viva Cádiz & 3 \\
\hline 3 & 20 minutos.es & 914 & 66 & Granada digital & 16 & 129 & El día.es & 3 \\
\hline 4 & La vanguardia.es & 500 & 67 & Diario Ibérico & 15 & 130 & Abertzale & 2 \\
\hline 5 & El periódico.com & 326 & 68 & Estrella digital.es & 14 & 131 & Alerta digital & 2 \\
\hline 6 & Público.es & 294 & 69 & Madridiario.es & 14 & 132 & Archipiélago noticias.com & 2 \\
\hline 7 & Europa press & 281 & 70 & PR noticias & 14 & 133 & Canarias 24 horas.com & 2 \\
\hline 8 & ADN.es & 252 & 71 & Diario crítico.com & 13 & 134 & Diario de Ferrol & 2 \\
\hline 9 & ABC.es & 246 & 72 & El periódico mediterráneo & 13 & 135 & Diario signo & 2 \\
\hline 10 & La voz de Galicia & 236 & 73 & La opinión de Tenerife & 13 & 136 & Diario de Jerez.es & 2 \\
\hline 11 & El plural.com & 228 & 74 & La voz de Asturias & 13 & 137 & Diario de Lanzarote.com & 2 \\
\hline 12 & El confidencial & 127 & 75 & La voz digital.es & 13 & 138 & Diario el faro Murcia & 2 \\
\hline 13 & Levante-EMV & 120 & 76 & D minorías & 12 & 139 & El correo.es de Andalucía & 2 \\
\hline 14 & El economista.es & 111 & 77 & ATB noticias.es & 11 & 140 & El boletín.es & 2 \\
\hline 15 & Libertad digital.es & 108 & 78 & Diario Córdoba online & 11 & 141 & El mercurio digital.es & 2 \\
\hline 16 & Periodista digital & 105 & 79 & El periodico Extremadura & 11 & 142 & Energía diario.com & 2 \\
\hline 17 & El correo digital & 91 & 80 & La región & 10 & 143 & Extremadura al dí@ & 2 \\
\hline 18 & Marca.com & 84 & 81 & 3i tercera información & 9 & 144 & Fuerteventura digital & 2 \\
\hline 19 & BBC mundo & 76 & 82 & Diario de Cádiz.es & 9 & 145 & Izaro news.com & 2 \\
\hline 20 & La razón.es & 72 & 83 & Diario de Ibiza.es & 9 & 146 & La crónica de Guadalajara & 2 \\
\hline 21 & Ideal.es & 70 & 84 & E-noticies.com & 9 & \begin{tabular}{|l|}
147 \\
\end{tabular} & La gran época.com & 2 \\
\hline 22 & La nueva España & 67 & 85 & Vila web & 9 & 148 & La mañana digital & 2 \\
\hline 23 & La república.es & 63 & 86 & Ávila digital.com & 8 & 149 & La democracia.es & 2 \\
\hline 24 & Canarias 7 & 58 & 87 & Canarias ahora.es & 8 & 150 & Madrid press.com & 2 \\
\hline 25 & Cotizalia & 55 & 88 & Diari de Tarragona.com & 8 & 151 & Rioja 2.com & 2 \\
\hline 26 & Cinco días & 52 & 89 & El progreso digital & 8 & 152 & Andalucía 24 horas & 1 \\
\hline 27 & Información.es & 51 & 90 & Diario de noticias Navarra & 7 & 153 & Atlántico & 1 \\
\hline 28 & La verdad.es & 51 & 91 & La opinión de Málaga.es & 7 & 154 & Auto sport.com & 1 \\
\hline 29 & Consumer.es & 50 & 92 & Rojo y negro & 7 & 155 & Ávila libre & 1 \\
\hline 30 & As.com & 45 & 93 & Extra confidencial.com & 6 & 156 & Avui & 1 \\
\hline 31 & Diario de Mallorca.es & 45 & 94 & La opinión de Zamora.es & 6 & \begin{tabular}{|l|}
157 \\
\end{tabular} & Bolsa press.es & 1 \\
\hline 32 & Expansión.com & 45 & 95 & Madrid digital & 6 & 158 & Ceuta al día.com & 1 \\
\hline 33 & Reuters & 45 & 96 & Sport.es & 6 & 159 & Ciber sur.com & 1 \\
\hline 34 & Soitu & 45 & 97 & Diario de noticias Gipuzkoa & 6 & 160 & Diario de Castilla & 1 \\
\hline 35 & Heraldo.es & 43 & 98 & Aragón digital.es & 5 & 161 & Diario salud & 1 \\
\hline 36 & Las provincias.es & 43 & 99 & Diario de avisos.com & 5 & 162 & Diario de Pontevedra & 1 \\
\hline 37 & Sur.es & 43 & 100 & Diario directo & 5 & 163 & Directe! cat & 1 \\
\hline 38 & El confidencial digital & 41 & 101 & Eldigital Castilla-La Mancha & 5 & 164 & El día de Valladolid digital & 1 \\
\hline 39 & El correo gallego.es & 40 & 102 & Mundo deportivo.es & 5 & 165 & El digital de Canarias & 1 \\
\hline 40 & Faro de Vigo.es & 40 & 103 & Ourense dixital.com & 5 & 166 & El día de Ciudad Real & 1 \\
\hline 41 & El semanal digital & 37 & 104 & Panorama actual.es & 5 & \begin{tabular}{|l|}
167 \\
\end{tabular} & El día de Córdoba.es & 1 \\
\hline 42 & Diario ti & 32 & 105 & Qué! & 5 & 168 & El diario exterior.com & 1 \\
\hline 43 & La flecha & 32 & 106 & Tribuna de Salamanca & 5 & \begin{tabular}{|l|}
169 \\
\end{tabular} & Es diari & 1 \\
\hline 44 & Hoy.es & 30 & 107 & ADN mundo.com & 4 & 170 & Es ahora.es & 1 \\
\hline 45 & Diario de León & 29 & 108 & AZ prensa.com & 4 & 171 & Girona notícies.com & 1 \\
\hline 46 & Diario de Sevilla.es & 29 & 109 & Diario de noticias Alava & 4 & 172 & Gomera verde.com & 1 \\
\hline 47 & Diario vasco.com & 26 & 110 & Diario del Alto Aragón & 4 & \begin{tabular}{|l|}
173 \\
\end{tabular} & Heraldo abierto & 1 \\
\hline 48 & El periódico de Aragón & 26 & \begin{tabular}{|l|l|}
111 \\
\end{tabular} & El ideal gallego & 4 & \begin{tabular}{|l|}
174 \\
\end{tabular} & Heraldo de Soria & 1 \\
\hline 49 & El norte de Castilla & 24 & 112 & El manifiesto.com & 4 & 175 & La nación & 1 \\
\hline 50 & El comercio digital.com & 23 & 113 & Europasur.es & 4 & 176 & La opinión digital & 1 \\
\hline 51 & Hispanidad & 23 & 114 & La opinión de Murcia.es & 4 & 177 & La voz de Marbella & 1 \\
\hline 52 & Diario siglo XXI & 22 & 115 & La provincia.es & 4 & 178 & La opinión Granada.es & 1 \\
\hline 53 & El diario montañés & 20 & 116 & Noticias.com & 4 & \begin{tabular}{|l|}
179 \\
\end{tabular} & Libertad balear.com & 1 \\
\hline 54 & La voz de Salamanca & 20 & \begin{tabular}{|l|l|}
117 \\
\end{tabular} & Málaga hoy.es & 4 & \begin{tabular}{|l|}
180 \\
\end{tabular} & Mallorca confidencial & 1 \\
\hline 55 & La opinión Coruña.es & 20 & 118 & Asturies.com & 3 & 181 & Nabarreria.com & 1 \\
\hline 56 & Diagonal periódico & 19 & 119 & Diario palentino digital & 3 & \begin{tabular}{|l|}
182 \\
\end{tabular} & Noticias cada día & 1 \\
\hline 57 & Metro & 19 & 120 & El imparcial & 3 & 183 & Noticias pyme & 1 \\
\hline 58 & Deia.com & 17 & 121 & Gaceta náutica.net & 3 & 184 & Novopress.info & 1 \\
\hline 59 & Diario de Navarra.es & 17 & 122 & Huelva información.es & 3 & 185 & Regió 7.cat & 1 \\
\hline 60 & Gaceta.es & 17 & 123 & La tribuna digital & 3 & \begin{tabular}{|l|}
186 \\
\end{tabular} & Telde actualidad.com & 1 \\
\hline 61 & Gara & 17 & 124 & Mallorca diario.com & 3 & 187 & Tenerife en línea & 1 \\
\hline 62 & Granada hoy.com & 17 & 125 & Minuto digital & 3 & 188 & Xornal.com & 1 \\
\hline 63 & Última hora digital & 17 & 126 & Noticias.info & 3 & & & \\
\hline
\end{tabular}

Tabla 1. Lista completa de medios de prensa en Menéame (enero 2007-abril 2008) 


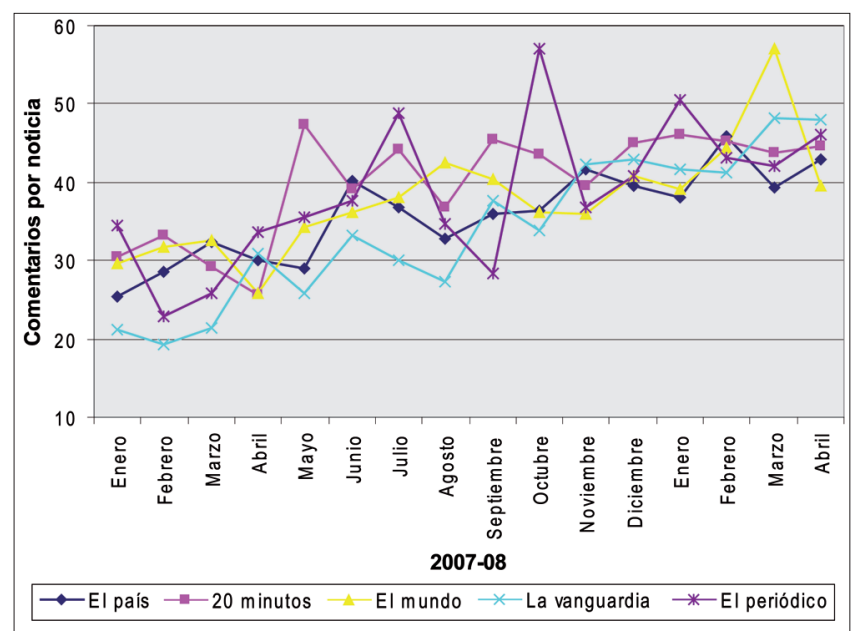

Figura 7. Evolución de comentarios por noticia en los cinco medios más representados

vanguardia, que ha pasado del último puesto en enero de 2007 al primero en abril de 2008. También es resaltable la caída de El mundo y la evolución irregular de El periódico.

En definitiva, sorprende cómo El país, con una cantidad de noticias superior al resto, no tiene una correspondencia en lo que a votos y comentarios se refiere.

\subsection{Distribución de los medios de prensa}

Teniendo en cuenta la totalidad de medios de prensa detectados, se obtiene la figura 8 con su distribución en función del número de noticias publicadas en 2007 en Menéame. La distribución es desigual: unos pocos medios publican muchas noticias, mientras que una gran cantidad de ellos (la "larga cola", vista aquí en una escala logarítmica) publica pocas.

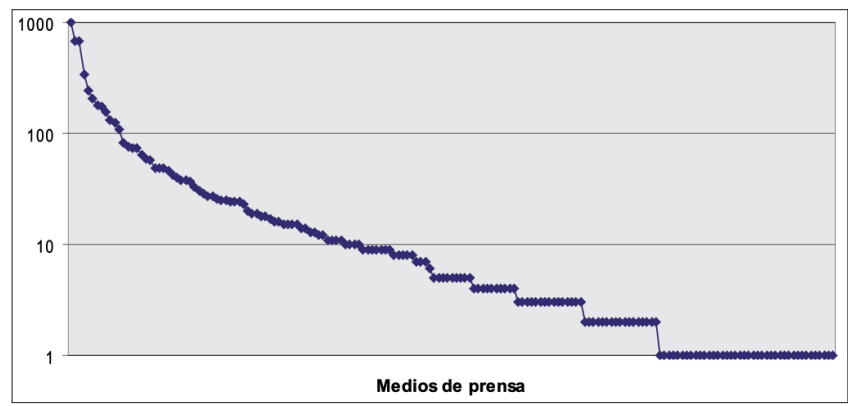

Figura 8. Distribución de los medios de prensa según el no de noticias

A partir de los datos que ofrece OJD Interactiva, se obtienen la figura 9 y la tabla 2 con la distribución de los medios de prensa en función del número de visitas diarias en 2007. En ella también aprecia la misma distribución desigual entre las webs.

El ranking de medios de prensa, en función del número de visitas proporcionado por OJD Interactiva, se detalla en la tabla 2, mientras que el ranking de $E G M$ (febrero 2007-noviembre 2007) se muestra en la tabla 3.

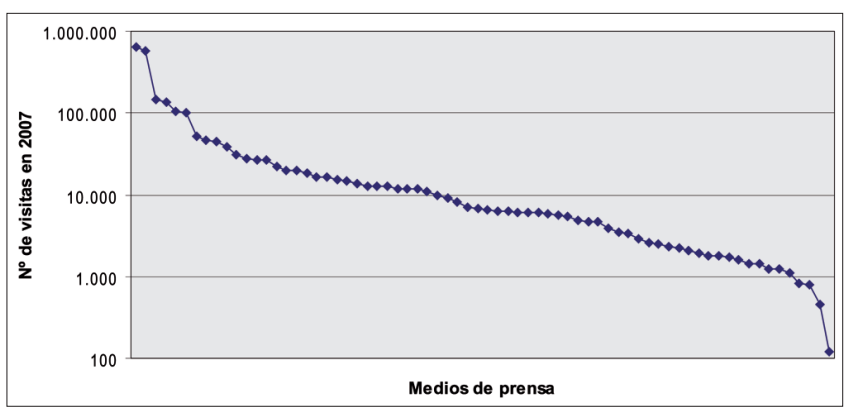

Figura 9. Distribución de los medios de prensa según número de visitas (en miles)

Fuente: OJD Interactiva

Destaca la diferencia entre ambos rankings, en especial la importante presencia de la prensa deportiva (frente a Menéame) y la ausencia de 20 minutos y $E l$ mundo en EGM.

\subsection{Discusión}

Como se comenta en la introducción del trabajo, el blog de Menéame ofrece estadísticas detalladas en cuanto a estudio de usuarios y noticias. Estos datos indican que la relación entre medios tradicionales y el resto es de $66 \%$ a $34 \%$ respectivamente.

\begin{tabular}{|l|c|}
\hline \multicolumn{1}{|c|}{ Medio } & Número de visitas \\
\hline Marca & 650.098 .125 \\
\hline El mundo & 564.260 .245 \\
\hline Sport & 145.857 .340 \\
\hline 20 minutos.es & 134.759 .029 \\
\hline ABC & 106.333 .444 \\
\hline Libertad digital & 99.504 .554 \\
\hline El periódico de Catalunya & 52.666 .156 \\
\hline Periodista digital & 47.047 .352 \\
\hline El confidencial & 44.685 .524 \\
\hline El correo digital & 38.401 .842 \\
\hline La voz de Galicia & 30.826 .102 \\
\hline La verdad & 27.709 .995 \\
\hline Expansión & 27.117 .306 \\
\hline La razón & 26.234 .824 \\
\hline Diario vasco electrónico & 22.495 .320 \\
\hline Ideal & 20.187 .880 \\
\hline La nueva España digital & 19.672 .323 \\
\hline Las provincias digital & 18.117 .809 \\
\hline Sur & 16.753 .915 \\
\hline El diario montañés & 16.634 .962 \\
\hline
\end{tabular}

Tabla 2. Ranking de medios por número de visitas (2007) Fuente: OJD Interactiva 


\begin{tabular}{|l|c|}
\hline \multicolumn{1}{|c|}{ Medio } & \multicolumn{1}{|}{$\begin{array}{c}\text { Visitantes únicos } \\
\text { durante 30 días } \\
\text { (en miles) }\end{array}$} \\
\hline Marca & 2.433 \\
\hline El país & 2.021 \\
\hline As & 1.268 \\
\hline El mundo deportivo & 680 \\
\hline Sport & 550 \\
\hline El periódico & 388 \\
\hline La vanguardia & 446 \\
\hline ABC & 305 \\
\hline El correo & 298 \\
\hline Expansión & 234 \\
\hline El norte de Castilla & 149 \\
\hline
\end{tabular}

Tabla 3. Ranking de medios (2007)

Fuente: Estudio General de Medios

Este último porcentaje coincide con los datos obtenidos en el inicio del presente estudio (un 33\% de noticias de medios de prensa a principios de 2007). No obstante hay que considerar tanto el diferente criterio a la hora de identificar medios tradicionales que han seguido, como el intervalo de la muestra.

Por otra parte el estudio realizado desde Es.ciudad aporta datos sobre rankings de medios y blogs desde el inicio de Menéame. A grandes rasgos los datos del presente artículo coinciden con los de ese estudio, exceptuando los relativos a El periódico de Catalunya.

También existe una concordancia respecto a la evolución de noticias que ofrece Es.ciudad de los diarios El país y El mundo para los primeros cuatro meses de 2007 (únicos en los que coincide el muestreo de datos).

Respecto al ranking de OJD Interactiva acerca del número de visitas a medios de prensa, sin entrar en la posible correlación entre éstas y el número de noticias publicadas en Menéame, no coincide con el obtenido en Menéame (asumiendo la ausencia en $O J D$ de diversos medios).

\section{Conclusiones}

1. La poca literatura científica existente sobre gestores sociales de noticias se centra en el estudio sociológico del comportamiento de los usuarios. No faltan por otro lado los datos estadísticos ni las noticias sobre el funcionamiento de estas webs.

2. Entre 1 de enero de 2007 y 30 de abril de 2008, el 36,4\% de noticias enviadas a Menéame procede de medios de prensa tradicionales. El número de noticias oscila en función de los meses, mostrando una tendencia al alza en los últimos meses.

3. Se detecta un crecimiento constante de la actividad de los usuarios de Menéame que se refleja en un aumento continuado tanto en el promedio de votos como de comentarios. Ello sin entrar a considerar la evolución del número de usuarios activos del sistema.

4. La distribución desigual de medios presentes en Menéame, en función del número de noticias aparecidas en él durante 2007, es similar a la detectada en $O J D$ Interactiva (para los medios que se someten a su análisis), en función del número total de visitas recibidas en el mismo período. De esta manera, se cumple en ambos casos la distribución de larga cola.

5. El medio de prensa más representado en $\mathrm{Me}$ néame es $E l$ país, al que le siguen a cierta distancia $E l$ mundo y 20 minutos y más alejados La vanguardia y El periódico de Catalunya.

6. En 2007 el ranking del número de noticias publicadas en Menéame no se corresponde con el de visitas por medio de OJD Interactiva ni con el de visitantes únicos de $E G M$, con una mayor presencia de prensa deportiva en estos últimos casos.

7. De cara a futuros estudios, tanto el sistema de medición de audiencias web como la amplitud de medios analizados podrían ser consideraciones fundamentales a la hora de intentar determinar si existe alguna correlación real entre un aumento del número de noticias en Menéame y un posible incremento del número de visitas en los datos de $O J D$, que permitiera comprobar el efecto de Menéame en la prensa digital.

\section{Notas}

1. http://bitelia.com/2007/05/02/digg-y-la-clave-para-desencriptar-los-hd$d v d$

2. Clones. En: Menéame: wiki. Consultado en: 04-04-08.

http://meneame.wikispaces.com/Clones

3. http://www.esciudad.com/meneame.html

4. http://www.lsi.us.es/ ffrosat/index.php/Ffrosat/TrabajoMeneame

5. http://blog.meneame.net/category/estadisticas/

\section{Bibliografía}

Cohen, M. Steven. "Have you reddit? Digg this". En: InfoToday, 2007, June, pp. 21-23.

Cohn, David. "Digg this". En: Columbia journalism review, 2007, Jan/Feb, v. 45, n. 5 , pp. $8-9$.

Dans, Enrique. “¿Vale dinero una 'personalidad online’?”. En: El blog de Enrique Dans. Consultado en: 04-04-08.

http://www.enriquedans.com/2006/07/vale-dinero-una-personalidadonline.html

"Digg vs. Slashdot (or, traffic vs. influence)". En: kottke.org: home of fine hypertext products. Consultado en: 04-04-08.

http://www.kottke.org/06/01/digg-vs-slashdot

Fernández, Luz. "El laberinto de la medición de audiencias 'online". En: El pais.com, 2007, diciembre. Consultado en: 17-04-08.

http://www.elpais.com/articulo/radio/television/laberinto/medicion/ audiencias/online/elpepugen/20071204elpepirtv_4/Tes

Guallar, Javier. "iMente: servicios de información de actualidad en línea". En: El profesional de la información, 2006, noviembre-diciembre, v. 15, n. 
6, pp. 426-435. Consultado en: 17-04-08.

http://eprints.rclis.org/archive/00007856/01/epi06_guallar_imente.pdf

Holahan, Catherine. "Digg goes deeper with social networking”. En: Business week online, 2007, September.

Lerman, Kristina. "Social information processing in social news aggregation". En: Internet computer, IEEE, 2007, v. 11, n. 6, pp. 16-28. Consultado en: $17-04-08$.

http://arxiv.org/PS_cache/cs/pdf/0703/0703087v2.pdf

Lerman, Kristina. "Social networks and social information filtering on Digg". En: arXiv:cs/0612046 [Submitted to the International conference on weblogs and social media].

http://arxiv.org/PS_cache/cs/pdf/0612/0612046v1.pdf

Lerman, Kristina. "User participation in social media: Digg study". En: Workshops of 2007 IEEE/WIC/ACM International conference on web intelligence and intelligent agent technology (WI-IAT 07).

http://arxiv.org/PS_cache/arxiv/pdf/0708/0708.2414v1.pdf

"Menéame y su falta de democracia”. En: Techlosofy: filosofía tecnológica. Consultado en: 04-04-08.

http://techlosofy.com/meneame-y-su-falta-de-democracia
Notess, Greg R. "Community filtering: Digg, Slashdot, and the social Web". En: Online, 2007, Jan/Feb, v. 31, n. 1, pp. 45-47.

Pons, Agustí. "MEO, Menéame engine optimization”. En: friky.com. Consultado en: 04-04-08.

http://friky.blogspot.com/2007/03/meo-meneame-engine-optimization.html

"Por qué me gusta y me disgusta Menéame". En: Alianzo: alianzo networks. Consultado en: 04-04-08.

http://blogs.alianzo.com/redessociales/2006/05/02/por-que-me-gusta-yme-disgusta-meneame

Salaverría, Ramón (coord.). Cibermedios: el impacto de internet en los medios de comunicación en España. Sevilla: Comunicación Social ediciones y publicaciones, 2005.

\section{Enrique Orduña-Malea, José-Antonio Ontalba-Rui-}

pérez, Universidad Politécnica de Valencia.

enorma@fiv.upv.es

joonrui@har.upv.es

\section{Máster Oficial Universitario}

\section{Contenidos y Aspectos Legales en la Sociedad de la Información}

\section{Objetivos}

Especializar a profesionales de la información en la gestión de contenidos a través de diferentes plataformas para todos los ámbitos de la sociedad. Ahondar y ampliar los conocimientos en Archivística, Biblioteconomía y Documentación con un enfoque dirigido a la aplicación de las tecnologías de la información en sus nuevos canales. Temas centrales son los contenidos audiovisuales.

\section{Especialidades}

- Información y Documentación: Gestión de contenidos multicanal.

Gestión de contenidos en nuevos formatos y distribuidos por distintos canales. Web 2.0, Redes sociales y Conocimiento colaborativo.

- E-Consulting en sociedad de la información. Planificación de sistemas de información, conocimiento de los procesos informativos en las organizaciones y de los contenidos y su distribución.

- Archivos y administración electrónica.

Gestión de los documentos electrónicos y sistemas de gestión documental. Administración electrónica.

\section{Estructura del Máster}

75 ECTS a impartir en un curso académico. Horario de tarde

Materias- 42,5 ECTS - se compone de asignaturas comunes y asignaturas de la especialidad que se escoja

Asignaturas de libre configuración curricular: 17,5 ECTS

Tesina fin de Máster:

15 ECTS

\section{Profesorado}

Este Máster será impartido por profesorado de diversas Universidades Nacionales así como profesionales de reconocido prestigio.

\section{Fechas}

Plazos de preinscripción:

Del 2 al 12 de Septiembre y del 1 al 8 de Octubre Plazos de Matrícula:

Del 23 al 30 de Septiembre

Del 20 al 24 de Octubre

\section{Información}

Secretaría del Departamento de Comunicación Audiovisual, Documentación e Historia del Arte (DCADHA) de la UPV.

Teléfono: 963877390

e-mail: dephar@upvnet.upv.es

Página Web:

http://www.upv.es/contenidos/PO/menu_494 351c.html

\section{Preinscripción}

http://www.upv.es/contenidos/PO/menu 49503 5c.html

Estos estudios dan acceso al programa de Doctorado

El master admite estudiantes titulados, tanto de nivel superior como de nivel medio (licenciados y diplomados)

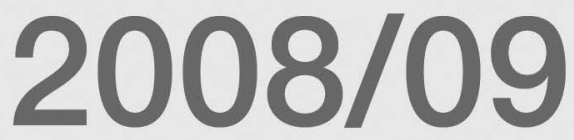

UNIVERSIDAD POLITECNICA DE VALENCIA
MINISTERIO

DE EDUCACIÓN Y CIENCIA
INSTITUTO

DE DISEÑO Y FABRICACIÓN 